www.nature.com/pj

\title{
Hyperbranched polyether core containing vegetable oil-modified polyester and its clay nanocomposites
}

\author{
Uday Konwar and Niranjan Karak
}

\begin{abstract}
Mesua ferrea L. seed oil-modified polyester with hyperbranched polyether core was prepared by the polycondensation technique, which involved carboxyl-terminated prepolymer of the monoglyceride of the oil, phthalic anhydride and maleic anhydride; hydroxyl of bisphenol-S and hyperbranched polyol of bisphenol-A and cyanuric chloride. The nanocomposites of this hyperbranched polyester with 0-5 wt\% nanoclay were prepared by an ex situ solution technique using strong mechanical shear force and ultrasonication. The degree of dispersion of the nanoclay in the polyester matrix and the resulting morphologies of nanocomposites were evaluated using $X$-ray diffraction, scanning electron microscopy and transmission electron microscopy. The studies revealed well-dispersed, intercalated or partially exfoliated nanocomposites. The effect of nanoclay on the mechanical, thermal and rheological behaviors of the nanocomposites was investigated. In the nanocomposite with 5 wt\% nanoclay, the tensile strength was doubled, the scratch hardness value was improved by $2 \mathrm{~kg}$ and the thermal degradation temperature was improved by $37^{\circ} \mathrm{C}$ when compared with the pristine polyester system. All results showed the potentiality of the prepared nanocomposites to be used as an advanced material.
\end{abstract}

Polymer Journal (2011) 43, 565-576; doi:10.1038/pj.2011.19; published online 16 March 2011

Keywords: exfoliation; Mesua ferrea L. seed oil; nanocomposites; polyester

\section{INTRODUCTION}

Ecological issues surrounding the development of new materials, the waste disposal of these materials and the exhaustion of non-renewable resources have increased the demand for renewable resources in industrial applications in contemporary times. ${ }^{1}$ Interesting research has been performed to substitute petroleum-based polymers with more innovative materials that can compete or even surpass the existing petroleum-based materials in terms of cost-performance and environmental friendliness. ${ }^{2}$ Natural vegetable oils are expected to be an ideal alternative chemical feedstock because of their many advantages, such as physical and chemical stability, aptitude to facile chemical modification, reduced toxicity, reduced risk for handling and transportation, possibility of recycling, renewability and biodegradability, and at the same time are available in large quantities at relatively low and stable prices, as well as susceptible to agricultural diversification and are environmentally benign in nature. These inexpensive triglycerides of fatty acids have been utilized extensively in various applications, such as coatings, inks, plasticizers, lubricants, resins and agrochemicals. ${ }^{3}$ However, conventional oil-based polymeric materials do not show the rigidity and strength for structural applications; ${ }^{4}$ hence, there is an urgent need to design a polymer from vegetable oils with a unique architectural structure.

Hyperbranched polymers are highly branched macromolecules with three-dimensional dendritic structures. They are the focus of interdisciplinary research and have received considerable attention over the past 20 years. The interest in such specially designed polymers is because of their unusual properties, such as high surface functionality, high solubility and reactivity, low viscosity, adequate compatibility with other materials and unique rheological behavior that provides an ideal outline to form films through efficient crosslinking. ${ }^{5-7}$ In addition, the properties of such polymers can be easily tailored by modifying the nature of their end groups with various functional groups, to satisfy the numerous demands of various applications. Thus, the synthesis and characteristics of novel threedimensional polymeric architectures have become the subject of increasing interest among polymer scientists and the focus of industrial research and applications. In this study, the properties of a hyperbranched polymer of a vegetable oil with unique structural features were investigated.

Among the different types of vegetable oil-based polymers, polyesters have shown great potential in many industrial applications. ${ }^{8-10}$ Therefore, the Mesua ferrea L. seed oil was used as one of the raw materials for the development of hyperbranched polyester, the potentialities of which were reflected from the literatures that were previously reported from the same laboratory. ${ }^{11-15}$

Further, nanostructured organic-inorganic composites have shown improved mechanical and thermal performances, chemical resistance, gas barrier and fire-retardant properties even at low loading of 
nanofillers in comparison with the pristine polymer system. ${ }^{16}$ Thus, forming a suitable nanocomposite using the proposed hyperbranched polyester may produce an advanced material with adequate performance. However, the ultimate performance improvement is dependent not only on the nature of the individual components but also on the degree of dispersion of the nanofillers and interfacial properties. ${ }^{17}$ Because organically modified montmorillonite (OMMT) has a high aspect ratio and interacts well with polyesters, it was investigated in this study. For proper dispersion, high-speed mechanical mixing and ultrasonication were used. The importance of such nanocomposites is reflected in recent literature as discussed in the following paragraph.

Haq et al. ${ }^{18}$ reported a bio-based nanocomposite that was composed of petroleum and vegetable oil-based polyester resin blend, with the addition of nanoclay for enhanced material properties. Bal et al. ${ }^{19}$ prepared nanocomposites of coconut oil-based polyester resins with organoclay, using an in situ method. The cured films with different ratios of melamine-formaldehyde and urea-formaldehyde resins exhibited improved properties. Bio-based nanocomposites from functionalized plant oils and layered silicates were reported by Lu et al. ${ }^{20}$ Although various vegetable oil-based polyester nanocomposites have been reported by various researchers, no report on Mesua ferrea L. seed oil-based hyperbranched polyester and OMMT nanocomposites exist to our knowledge.

Therefore, the main objective of this study is to develop a vegetable oil-based hyperbranched polyester resin and its nanocomposites with OMMT that have interesting structural features. The preparation of hyperbranched polyester from Mesua ferrea L. seed oil was reported in this paper; in addition, the mechanical, thermal, rheological and chemical properties of hyperbranched polyester and its nanocomposites upon loading of OMMT were analyzed.

\section{EXPERIMENTAL PROCEDURE}

\section{Materials}

Mesua ferrea L. seed oil was extracted from its seeds (Sivasagar, Assam). Phthalic anhydride (Merck, Hohenbrunn, Germany), maleic anhydride (Merck), 4,4'-sulfonyldiphenol (Bisphenol-S, BPS, Merck), glycerol (Merck) and lead monoxide (S.D. Fine Chemical Ltd, Mumbai, India) were used without any further purification. Hyperbranched polyether (HBPE, $M_{\mathrm{w}}=3200 \mathrm{~g} \mathrm{~mol}^{-1}$ ) was prepared using the method reported earlier. ${ }^{21}$ Cyanuric chloride (Merck) and bisphenol-A (BPA, S.D. Fine Chemical Ltd) were recrystallized from chloroform and toluene, respectively, before use. $N, N^{\prime}$ Dimethylacetamide (Merck, Mumbai, India), N,N'-dimethylformamide (Merck), xylene (Merck) and methanol (Merck) were purified by conventional methods before use. Sodium metal (Merck) was used as received. Nanoclay (Nanomer ${ }^{\mathrm{R}}$ I.30E, montmorillonite clay modified with $25-30 \mathrm{wt} \%$ octadecylamine (OMMT, Sigma-Aldrich, Taufkirchen, Germany)) was used as the source of the nanomaterial. BPA-based diglycidyl ether epoxy resin (GY 250), diethylene triamine and dimer acid-based poly(amido amine) hardener (HY 840, from Ciba Geigy, Mumbai, India) were used as curing agents as supplied. All other reagents were of reagent grade and used without further purification.

\section{Instruments and methods}

The purified oil was obtained by the solvent soaking method from matured Mesua ferrea L. seeds, followed by the alkali refining purification technique. ${ }^{11}$ The Fourier transform infrared (FTIR) spectrum of the HBPE-modified polyester resin was recorded on an FTIR spectrometer (Nicolet Impact 410, Madison, WI, USA), using a $\mathrm{KBr}$ pellet. The ${ }^{1} \mathrm{H}$ and ${ }^{13} \mathrm{C}$ nuclear magnetic resonance (NMR) spectra of the HBPE and the polyester resin were recorded using an FT-NMR spectrometer (model JNM-ECS, $400 \mathrm{MHz}$, JEOL, Tokyo, Japan) and $\mathrm{CDCl}_{3}$ as solvent. An ultrasonic processor (UP200S, Hielscher, Teltow, Germany) was used at fixed amplitude (60\%) for a half cycle to improve the dispersion of OMMT. X-ray diffraction (XRD) studies were carried out at a scanning rate of $0.05^{\circ} \mathrm{min}^{-1}$ over the range of $2 \theta=0-60^{\circ}$, using an X-ray diffractometer (Rigaku Miniflex, Tokyo, Japan). The thermogravimetric analysis of the cured nanocomposites was carried out using a thermal analyzer (Thermogravimetric analysis 50, Shimadzu, Tokyo, Japan) under nitrogen flow at a rate of $30 \mathrm{ml} \mathrm{min}^{-1}$ and a heating rate of $10^{\circ} \mathrm{C} \mathrm{min}-1$. Gloss, scratch hardness and impact resistance of the nanocomposite films were measured using a digital mini gloss meter (Sheen Instrument Ltd, Surrey, UK), a scratch hardness tester (Sheen Instrument Ltd) and a falling weight impact tester (SC Dey, Kolkata, India), respectively, according to the standard test methods. ${ }^{11}$ The rheological study of the polyester resin and its nanocomposites was performed using a rheometer (CVO100, Malvern, UK). The relationships of viscosity against time and viscosity against temperature were measured at constant stress $(200 \mathrm{~Pa})$ under single shear. The variation of shear viscosity with temperature was measured in the range of $25-100^{\circ} \mathrm{C}$ at a shear stress of $70 \mathrm{~Pa}$. The surface morphology of the nanocomposite films was studied using a scanning electron microscope (SEM, model JSM-6390LV, JEOL) after coating the surface of the fractured sample with platinum. The sizes and distributions of OMMT layers in the nanocomposites were studied using a transmission electron microscope (TEM, model JEMCXII, JEOL) operated with an accelerating voltage of $100 \mathrm{kV}$ and equipped with a digital camera. The dilute polymer nanocomposite solution was transferred to a carbon-coated copper grid. None of the sections was stained with heavy metal before imaging because the contrast between the fillers and the polymer matrix was sufficient. A universal testing machine (model Zwick Z010, Ulm, Germany) was used to measure the tensile strength and elongation at break of the nanocomposite films with a $10-\mathrm{kN}$ load cell and at a jaw separation speed of $50 \mathrm{~mm} \mathrm{~min}^{-1}$ under ambient conditions. Following the American society for testing and materials (ASTM) D593-67 method, ${ }^{11}$ different chemical environments were applied to the cured nanocomposite films to investigate the chemical resistance.

\section{Preparation of the sodium salt of BPA}

The sodium salt of BPA was prepared by treating the latter with metallic sodium in 'super dry' methanol, according to the procedure reported earlier. ${ }^{21}$

\section{Preparation of HBPE}

The HBPE was prepared following the procedure reported earlier. ${ }^{21}$ Briefly, cyanuric chloride $\left(2.73 \mathrm{~g}, 0.0148 \mathrm{~mol}\right.$ ) solution (with $N, N^{\prime}$-dimethylacetamide as a solvent) was added dropwise to the sodium salt of BPA solution with the same solvent $(6.034 \mathrm{~g}, 0.022 \mathrm{~mol})$ under nitrogen atmosphere, with constant stirring at room temperature $\left(27^{\circ} \mathrm{C}\right)$ for $2 \mathrm{~h}$. The reaction was subsequently continued at $70{ }^{\circ} \mathrm{C}$ for $2 \mathrm{~h}$, followed by heating at $137 \pm 2{ }^{\circ} \mathrm{C}$ for another $8 \mathrm{~h}$. The sticky mass was obtained by pouring the filtrate of the reaction mixture dropwise in ice water and purified by re-precipitation with acetone and water. The product was finally washed with toluene to remove any unreacted cyanuric chloride and dried under vacuum at $60^{\circ} \mathrm{C}$ for two days. The yield of the product was $65 \% .{ }^{1} \mathrm{H}$ NMR $\left(\mathrm{CDCl}_{3}\right) \delta: 7.23(\mathrm{Ph}, 16 \mathrm{H}), 7.07-6.7(\mathrm{Ph}, 16 \mathrm{H})$, $5.21(\mathrm{OH}, 1 \mathrm{H})$ and $1.62-1.60\left(\mathrm{CH}_{3}, 24 \mathrm{H}\right)$ p.p.m. ${ }^{13} \mathrm{C} \mathrm{NMR}\left(\mathrm{CDCl}_{3}\right) \delta: 171$ $(\mathrm{Ph}), 172(\mathrm{Ph}), 173(\mathrm{Ph}), 153(\mathrm{Ph}), 149-148(\mathrm{Ph}), 143(\mathrm{Ph}), 142(\mathrm{Ph}), 128$ (Ph), $127(\mathrm{Ph}), 120(\mathrm{Ph}), 114.6(\mathrm{Ph}), 42\left(-\mathrm{C}_{\text {ter }}\right)$ and $31.4\left(\mathrm{CH}_{3}\right)$ p.p.m.

\section{Preparation of polyester resin}

First, a carboxyl-terminated prepolymer was prepared using a method reported previously. ${ }^{11}$ Briefly, $42.09 \mathrm{~g}$ ( $\left.0.12 \mathrm{~mol}\right)$ of monoglyceride was reacted with $6.27 \mathrm{~g}$ $(0.064 \mathrm{~mol})$ of maleic anhydride and $14.22 \mathrm{~g}(0.096 \mathrm{~mol})$ of phthalic anhydride to prepare the carboxyl-terminated prepolymer. Further, $15.65 \mathrm{~g}$ of the prepolymer was reacted with $2.5 \mathrm{~g}(0.01 \mathrm{~mol})$ of BPS and $0.25 \mathrm{~g}$ of HBPE ( $10 \%$ the amount of BPS) in $N, N^{\prime}$-dimethylformamide $(25 \mathrm{ml})$ at $145-150^{\circ} \mathrm{C}$ for $3.5 \mathrm{~h}$ under nitrogen atmosphere, with continuous stirring. The temperature of the reaction was then raised slowly to $220^{\circ} \mathrm{C}$ and finally cooled to room temperature to obtain the desired viscous resin. The yield of the product was $\sim 90-95 \% .{ }^{1} \mathrm{H}$ NMR $\left(\mathrm{CDCl}_{3}\right)$

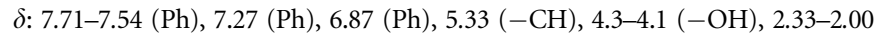
$(-\mathrm{CH}), 1.60\left(-\mathrm{CH}_{2}-\right), 1.29-1.25\left(-\mathrm{CH}_{2}-\right)$ and $0.92-0.89\left(\mathrm{CH}_{3}\right)$ p.p.m.

\section{Preparation of nanocomposites}

Different dose levels (1, 2.5 and $5 \mathrm{wt} \%$ ) of OMMT were dispersed in xylene by mechanical shearing for $30 \mathrm{~min}$, followed by ultrasonication for $10 \mathrm{~min}$ under the conditions specified in the Experimental procedure section. The desired 
amount of dispersed OMMT $(1,2.5$ and $5 \mathrm{wt} \%)$ was then mixed with polyester resin by vigorous mechanical stirring, followed by ultrasonication for $30 \mathrm{~min}$. After sonication, calculated amounts of epoxy resin (the polyester to epoxy resin ratio was $60-40 \mathrm{wt} \%)$ and poly(amido amine) hardener (25\% of the resin) were mixed to form the thermoset matrix. Finally, the mixture was degassed for $30 \mathrm{~min}$ under vacuum to remove all bubbles. The nanocomposites were denoted as PNC1, PNC2.5 and PNC5 corresponding to the clay content of $1,2.5$ and $5 \mathrm{wt} \%$, respectively; the pristine system without clay was denoted as PE.

\section{Curing study}

After complete removal of the solvent and the bubbles, the nanocomposite mixtures were cast on mild steel plates $(150 \times 50 \times 1.60 \mathrm{~mm})$ and glass plates $(75 \times 25 \times 1.75 \mathrm{~mm})$ for further study. The as-cast films were then dried in vacuum desiccators overnight at room temperature, followed by curing at $120^{\circ} \mathrm{C}$ for further study. The curing time was estimated using the hard drying time of the 40 - to $50-\mu \mathrm{m}$-thick films (measured with the Pen tester made by Sheen instrument Ltd) cast on glass plates. The hard drying time was the minimum time required for curing the film at the specified temperature by measuring the resistance to indentation using an indenter (pin), followed by a swelling test.

\section{Swelling test}

The swelling test was performed by immersing weighted films in xylene. After $48 \mathrm{~h}$, the weight of the swelled films was measured. Swelling value (\%) was determined by the difference in weight between the dried film and the swelled film as follows:

$$
\text { Swelling }(\%)=\left[\left(W_{s}-W_{\mathrm{d}}\right) / W_{\mathrm{d}}\right] \times 100
$$

where $W_{\mathrm{s}}$ and $W_{\mathrm{d}}$ are the weights of the swelled film and the dried film, respectively.

\section{RESULTS AND DISCUSSION}

\section{Preparation of the HBPE}

The HBPE was prepared by reacting cyanuric chloride with the sodium salt of BPA, using the $\mathrm{A}_{2}+\mathrm{B}_{3}$ approach. Because the reactivities of the three chlorine atoms of the triazine moiety are different, the reaction was carried out at three different temperatures through three steps. The sodium salt of BPA was used because it has a higher reactivity than BPA. The polymer synthesis details were reported earlier. $^{21}$

\section{Preparation of the polyester resin}

The polyester resin was prepared from the carboxyl-terminated prepolyester of Mesua ferrea L. seed oil, BPS and HBPE. The HBPE was used as a core molecule to enhance different properties of the polyester, such as processability, thermal stability and chemical resistance due to its hyperbranched structure with the s-triazine moiety. The renewable resource-based prepolyester provided the structure based on the composition of the oil, whereas BPS helped the thermostability and rigidity of the polyester. The high yield of the resin (90-95\%) may be attributed to the use of a judicious amount of solvent that facilitated the homogenization of the reactants. The proposed reaction scheme of the prepared polyester with HBPE core is shown in Scheme 1.

\section{Characterization of HBPE}

FTIR analysis. The prepared HBPE showed the important bands such as the aromatic ether band at $1240 \mathrm{~cm}^{-1}$ and other characteristic absorbance bands such as the phenyl group and the methyl group of the BPA moiety at 2960, 1504, 826 and $552 \mathrm{~cm}^{-1}$ in the FTIR spectrum (not shown), as reported earlier. ${ }^{21} \mathrm{~A}$ strong band observed at $3354 \mathrm{~cm}^{-1}$ was due to the presence of the $\mathrm{H}$-bonded surface hydroxyl groups of the polyether.
${ }^{1} H$ NMR analysis. The detailed structure of the polyether was characterized by NMR spectroscopy. The ${ }^{1} \mathrm{H}$ NMR spectrum of the polyether is shown in Figure 1. The peaks at $\delta=1.60-1.62$ p.p.m. were due to the $-\mathrm{CH}_{3}$ protons of the BPA moiety. The broad peak at $\delta=5.21$ p.p.m. was due to the surface $-\mathrm{OH}$ group of the polyether. The peaks at $\delta=6.7-7.07$ p.p.m. were due to the $-\mathrm{CH}$ aromatic protons of the BPA moiety. The peak arising at $\delta=7.23$ p.p.m. was due to the protons of the solvent $\left(\mathrm{CDCl}_{3}\right)$ used.

${ }^{13} \mathrm{C}$ NMR analysis. Figure 2 shows the different peaks observed for different $\mathrm{C}$ atoms in the ${ }^{13} \mathrm{C}$ NMR spectrum of the prepared polyether. The peaks for different $\mathrm{C}$ atoms of the BPA moiety labeled a, b, c, d, e, $\mathrm{f}, \mathrm{g}, \mathrm{h}, \mathrm{i}$ and $\mathrm{j}$ are found at $\delta=148-149,120,127,142,42,31.4,143$, $128,114.6$ and 153 p.p.m., respectively, in the probable structure of the polyether (Figure 2). In addition to these peaks, three other peaks were observed at $\delta=171,172$ and 173 p.p.m. that may be due to dendritic, linear and terminal units of the polyether structure, respectively. Using ChemDraw Ultra 8.0 software (CambridgeSoft Corporation, Campridge, MA, USA), these peaks were compared with low-molecular-weight model compounds containing the theoretical dendritic, linear and terminal units. The model compounds showed the peaks for dendritic, linear and terminal units at $\delta=181.8$, 185.9 and 187 p.p.m., respectively. Similar peak assignments of s-triazine containing aliphatic polymer have been reported. ${ }^{22}$ The peak found at $\delta=77.3$ p.p.m. was also due to the solvent $\left(\mathrm{CDCl}_{3}\right)$ used.

Degree of branching. The structural perfection of the hyperbranched polymers is usually characterized by determining their degree of branching (DB), which was determined here using Frechet's equation, $\mathrm{DB}=(D+T) /(D+T+L)$, where $D, T$ and $L$ refer to the number of dendritic, terminal and linear units in the structure of the polymer, respectively. Experimentally, DB was determined from ${ }^{13} \mathrm{C}$ NMR spectroscopy by comparing the integration of the peaks for the respective units in the polyether (Figure 2 ). The integration values of the peaks for $D, T$ and $L$ in the polyether were 6.08, 13.8 and 8.7, respectively, and hence the $\mathrm{DB}$ value for the polyether was 0.69 . The value of DB was close to $0,0.5$ and 1.0 for the linear, hyperbranched and dendrimer structure, respectively. Thus, the polyether exhibited a highly branched structure rather than a linear structure.

\section{Characterization of polyester resin}

FTIR analysis. The FTIR spectrum of the polyester resin (Figure 3) indicated the presence of an important linkage, such as ester groups $\left(1733 \mathrm{~cm}^{-1}\right)$ and olefinic double bonds. Other characteristic bands present in the FTIR spectrum for HBPE, such as bands for the phenyl group and the methyl group of the BPA moiety also appeared at 1507, 834 and $559 \mathrm{~cm}^{-1}$, in addition to the aromatic ether linkage at $1248 \mathrm{~cm}^{-1}$. These revealed the formation of a polyester resin through the acid group of the prepolyester with the $-\mathrm{OH}$ groups of BPS and HBPE. A band for the unsaturation of maleic anhydride moiety at $1645 \mathrm{~cm}^{-1}$ was also observed in the resin. The band appeared at $3397 \mathrm{~cm}^{-1}$, which indicated the presence of the hydroxyl group in the polyester resin. The bands at 2926 and $2858 \mathrm{~cm}^{-1}$ represented the aliphatic - $\mathrm{C}-\mathrm{H}$ symmetric and asymmetric stretching, respectively, of the polyester resin.

${ }^{1} \mathrm{H}$ NMR analysis. The ${ }^{1} \mathrm{H}$ NMR spectrum of the vegetable oilmodified polyester with HBPE core is shown in Figure 4. In addition to the peaks obtained for the HBPE, the other peaks observed for different proton environments were as follows: the peaks for the protons of terminal methyl group of the fatty acid chains were 


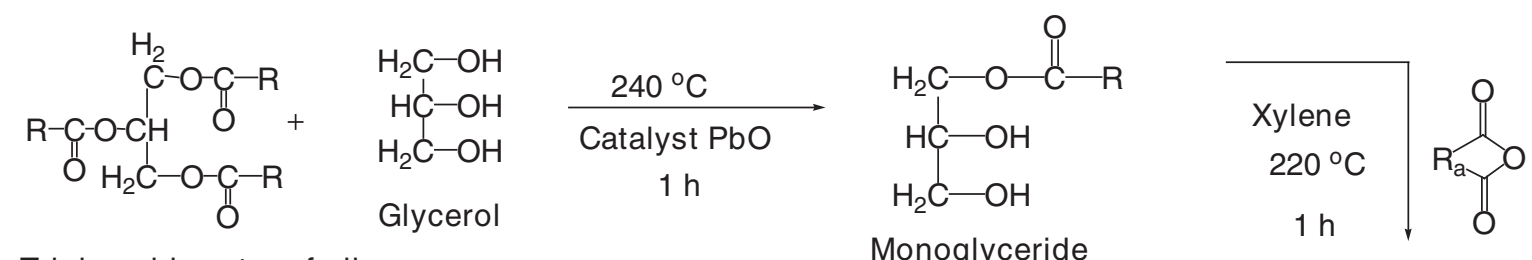

Triglyceride ester of oil<smiles>[R]C(=O)OCC(C)OC(=O)CC(=O)OC</smiles>
Prepolymer<smiles>[R5]C=CC[Hg]c1ccc(C(C)C)cc1</smiles>

Monoglyceride

$\mathrm{HOOC} \sim \mathrm{m} \sim \mathrm{COOH}$<smiles></smiles>
$220^{\circ} \mathrm{C} \mathrm{OH}+$

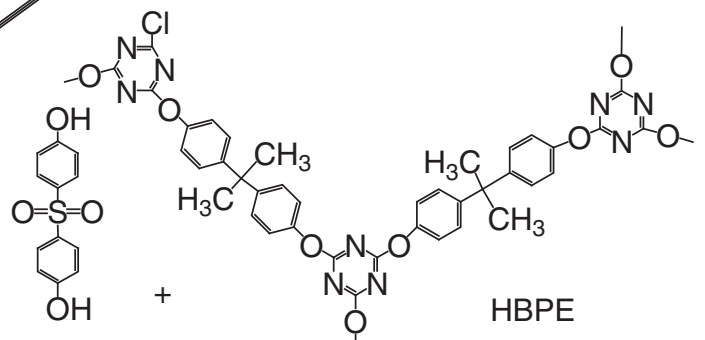
BPS<smiles>c1ccccc1</smiles><smiles>CC(C)C</smiles><smiles>c1ccccc1</smiles>
$\mathrm{N}^{2} \mathrm{~N}$ $\mathrm{Cl}^{2} \mathrm{~N}^{\mathrm{C}} \mathrm{Cl}$<smiles>CCC(C)Oc1nc(Oc2ccc(C(C)(C)c3ccc(OC(=O)CC(=O)OC4CCC(S(=O)(=O)C5CCC(OC)CC5)CC4)cc3)cc2)nc(OC(C)CC)n1</smiles><smiles>Cc1ccc(C(C)(C)C2CCC(C)CC2)cc1</smiles> 

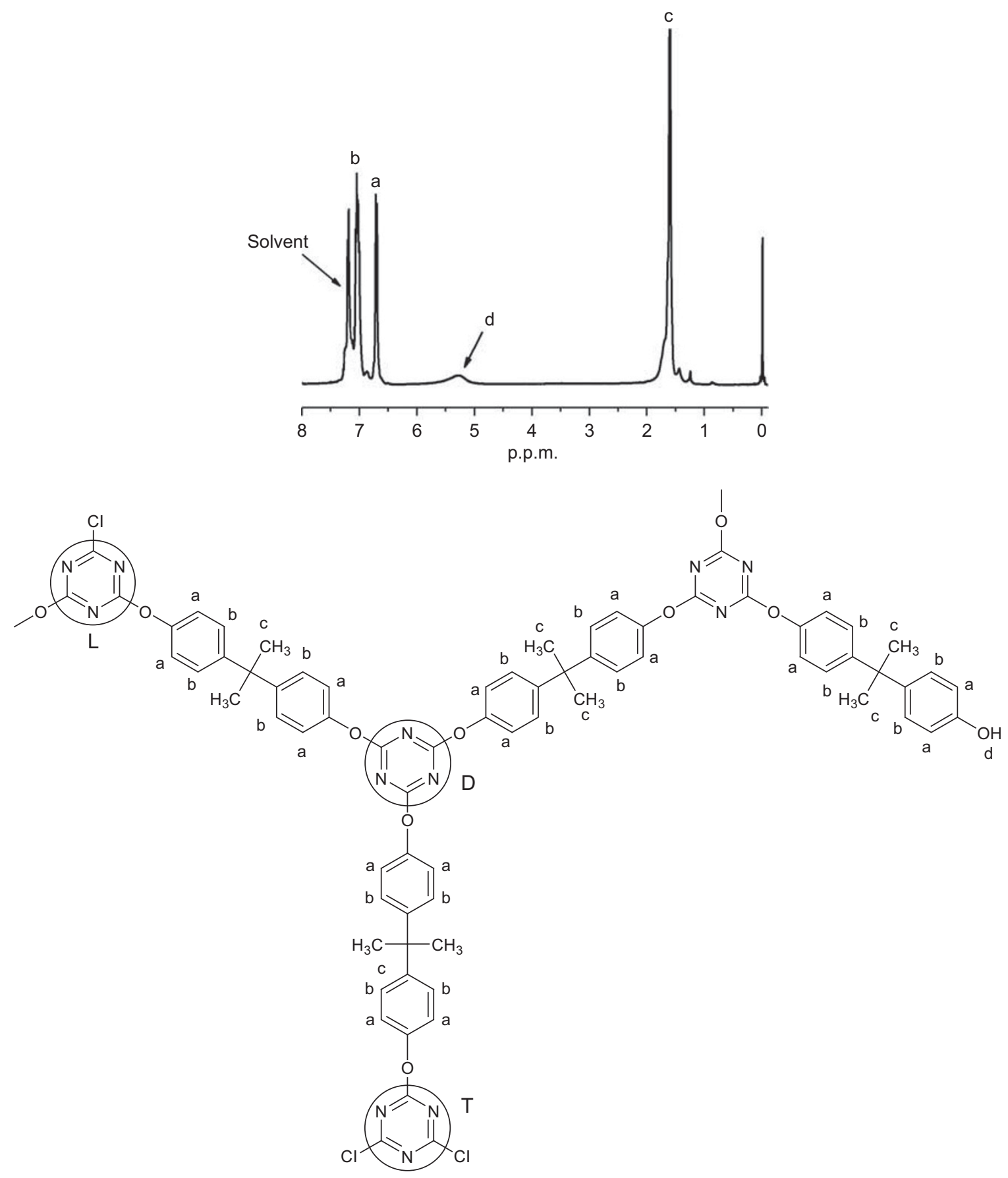

Figure $1^{1} \mathrm{H}$ nuclear magnetic resonance spectrum of hyperbranched polyether.

found at $\delta=0.89-0.92$ p.p.m.; the peaks for protons of $-\mathrm{CH}_{2}-$ moiety attached next to the terminal methyl group appeared at $\delta=1.60$ p.p.m.; and the peaks observed at $\delta=1.25-1.29$ p.p.m. were due to the protons of all the internal $-\mathrm{CH}_{2}-$ groups present in the fatty acid chains. For the protons of unsaturated carbons, peaks appeared at $\delta=5.33$ p.p.m. The large, broad peak observed at $\delta=4.1-4.3$ p.p.m. was due to the hydroxyl group on the surface of the polyester, which was exchangeable with deuterium, as clearly seen from proton NMR. The peaks due to the aromatic protons of BPA moiety and BPS moiety were observed at $\delta=6.87$ p.p.m. and $\delta=7.27$ p.p.m., respectively. The aromatic protons of phthalic anhydride and protons for maleic anhydride units were found at $\delta=7.54-7.71$ p.p.m. and at $\delta=2.00-2.33$ p.p.m., respectively. However, it was rather difficult to assign the exact structural characterization and to assign all peaks from the NMR spectrum due to the very complex structure of the synthesized polyester, which contains long-chain fatty acids along with other natural components.

\section{Preparation of nanocomposites}

The nanocomposites were fabricated to enhance properties such as the mechanical, thermal and chemical resistances of the vegetable oilbased polyester resin. The structure and the degree of dispersion of the OMMT in the polyester matrix had an important role in achieving the 

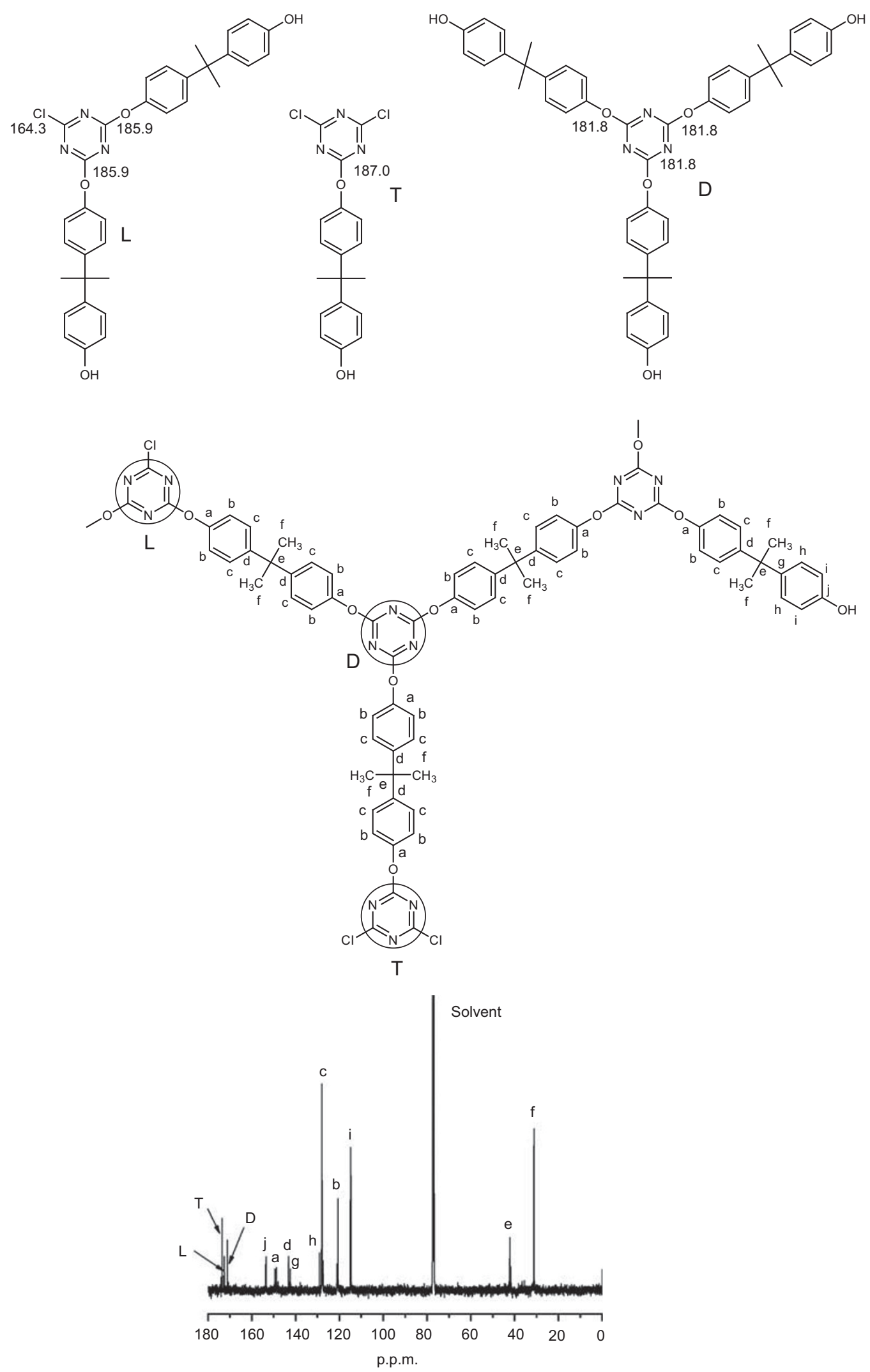

Figure $2{ }^{13} \mathrm{C}$ spectrum of hyperbranched polyether. 


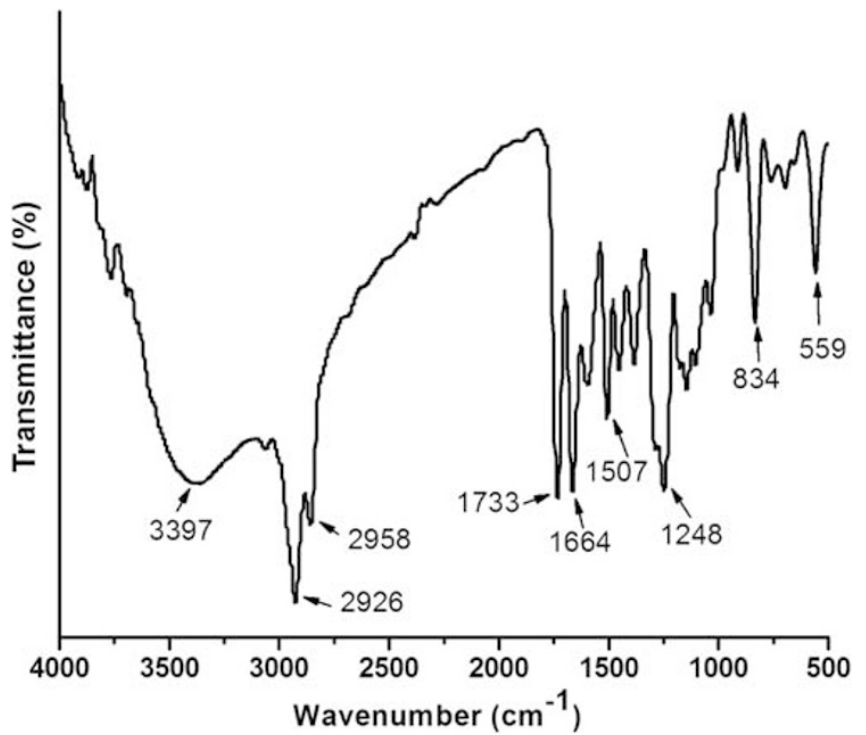

Figure 3 Fourier transform infrared spectrum of the vegetable oil-modified polyester with hyperbranched polyether core.

desired properties. Proper dispersion of the OMMT was achieved with high shear forces by vigorous mechanical mixing and ultrasonication. Ultrasonication can produce tiny bubbles, which can collapse violently and release significant energy that can be used to exfoliate OMMT layers. ${ }^{23}$ This prolonged force promoted the penetration of polymer chains into the galleries between the silicate layers. This in turn resulted in the delaminated/exfoliated structure, in which the individual OMMT layers were completely separated and dispersed randomly in the polyester matrix. Here, the carboxyl groups of the polyester could easily interact with the hydroxyl groups of OMMT through H-bonding or other polar-polar interactions.

\section{Characterization of nanocomposites}

XRD analysis. Figure 5 shows the XRD patterns of the OMMT and the nanocomposite samples fabricated with different OMMT contents. The characteristic peak of the pristine OMMT appeared at $2 \theta=4.15^{\circ}$ with corresponding interlayer spacing $\mathrm{d}=2.12 \mathrm{~nm}$, calculated from the Bragg's equation (Table 1). For nanocomposite PNC1, this peak was shifted to $2 \theta=3^{\circ}(d=2.94 \mathrm{~nm})$, suggesting that the OMMT was swollen because of the polymer chains, which led to increased $d$-spacing. For PNC2.5, the above peak was further shifted toward a lower angle of $2 \theta=2.12^{\circ}(d=4.16 \mathrm{~nm})$. In general, a larger interlayer spacing should be advantageous in the intercalation of polymer chains. It should also lead to easier dissociation of the OMMT layers, resulting in nanocomposites with better OMMT dispersions. ${ }^{24}$ Thus, intercalation of the OMMT layers by the polyester chains was obtained. The PNC5 sample showed no peak for OMMT, although a broad peak at $2 \theta=19^{\circ}$ in the XRD diffractogram was attributed to the amorphous nature of the pristine polyester resin; this broad peak was present in all three nanocomposites. The absence of the characteristic peak of OMMT in the PNC5 sample may be due to the exfoliation of the OMMT layers by the polyester chains. This also suggested the disordering and loss of structural regularity of the OMMT layers. Although the exfoliated state of the OMMT layer can be estimated by the absence of the [001] peak reflection, it was difficult to reach a conclusion about the actual structure obtained from XRD alone. The improved thermo-mechanical characteristics were attributed to the dispersion of OMMT in the polyester matrix.

SEM analysis. The surface morphologies of the nanocomposite films were depicted in the SEM images. In the SEM micrographs (Figure 6), wire-like structures due to OMMT layers were observed. These OMMT layers were uniformly distributed throughout the polyester matrix in all of the nanocomposites ( $1-5 \mathrm{wt} \%)$. The OMMT layers were also found to increase with the increasing OMMT loading. This homogeneous distribution of OMMT was attributed to the interaction of the $-\mathrm{OH}$ groups of OMMT with the ester groups of the polyester through H-bonding or other polar-polar interactions.

TEM analysis. A representative TEM image of the polyester nanocomposite is shown in Figure 7. The TEM image of the nanocomposite was taken to visually evaluate the degree of intercalation, exfoliation and aggregation of the clay clusters and confirm the XRD results. The TEM micrograph of the nanocomposite (PNC5) showed homogeneous dispersion and an exfoliated structure, supporting the XRD findings. Thus, after formation of nanocomposites, the individual OMMT layers were found to be disintegrated or partially exfoliated and well dispersed in the polyester matrix. The probable reason for this excellent distribution is the strong interaction between the polar carboxylic ester groups of the hyperbranched polyester and the $-\mathrm{OH}$ group of OMMT. The highly branched, globular, high surface functionality of the hyperbranched polyester also contributed to the strong interactions.

\section{Performance characteristics of the nanocomposites}

The performance characteristics of the vegetable oil-modified polyester with HBPE core and its nanocomposites are given in Table 2. The tensile strength of PNC5 improved to a value of $5.72 \mathrm{~N} \mathrm{~mm}^{-2}$ compared with the pristine polymer. This enhancement could be attributed to the interaction of immobilized or partially mobilized polymer chains with OMMT clay. Again, the high aspect ratio and large surface area of the nanoclay contributed to the adhesion between the polymer molecules and the OMMT layers. As a result, the reinforcing phase of the nanocomposite was efficient at bearing high stress ${ }^{25}$ and contributed to the improvement in tensile strength. Motion of the polymer chains was effectively constrained because of stronger interactions between OMMT layers and the polymer molecules associated with a larger contact surface. ${ }^{4}$ With the increase in the amount and dispersion of OMMT layers, the load-bearing capacity also improved. However, the elongation at break of the nanocomposites decreased from 102.21 to $47.75 \%$ with the increase of OMMT loadings from 0 to $5 \mathrm{wt} \%$. This was because the OMMT layers restricted the motion of the polymer chains.

The gloss values of the nanocomposites were enhanced with the increase of OMMT loading, as shown in Table 2. All components of polyester resin, epoxy resin, hardener and OMMT were compatible with each other, as reflected in these gloss values. In the nanocomposites, improved crosslinking of the cured films occurred as OMMT loading increased from 0 to $5 \mathrm{wt} \%$. This enhanced crosslinking formed smooth surfaces that improved the gloss values of the nanocomposites. In addition, large amounts of light were reflected from the smooth surface of the films as the OMMT content increased, which also increased the gloss value. A significant improvement in scratch hardness was observed in the nanocomposites reinforced with 1, 2.5 and $5 \mathrm{wt} \%$ OMMT. The effective network formed through the physical crosslink after inclusion of OMMT restricted the indentation and enhanced the scratch hardness of the films. The impact resistance of 

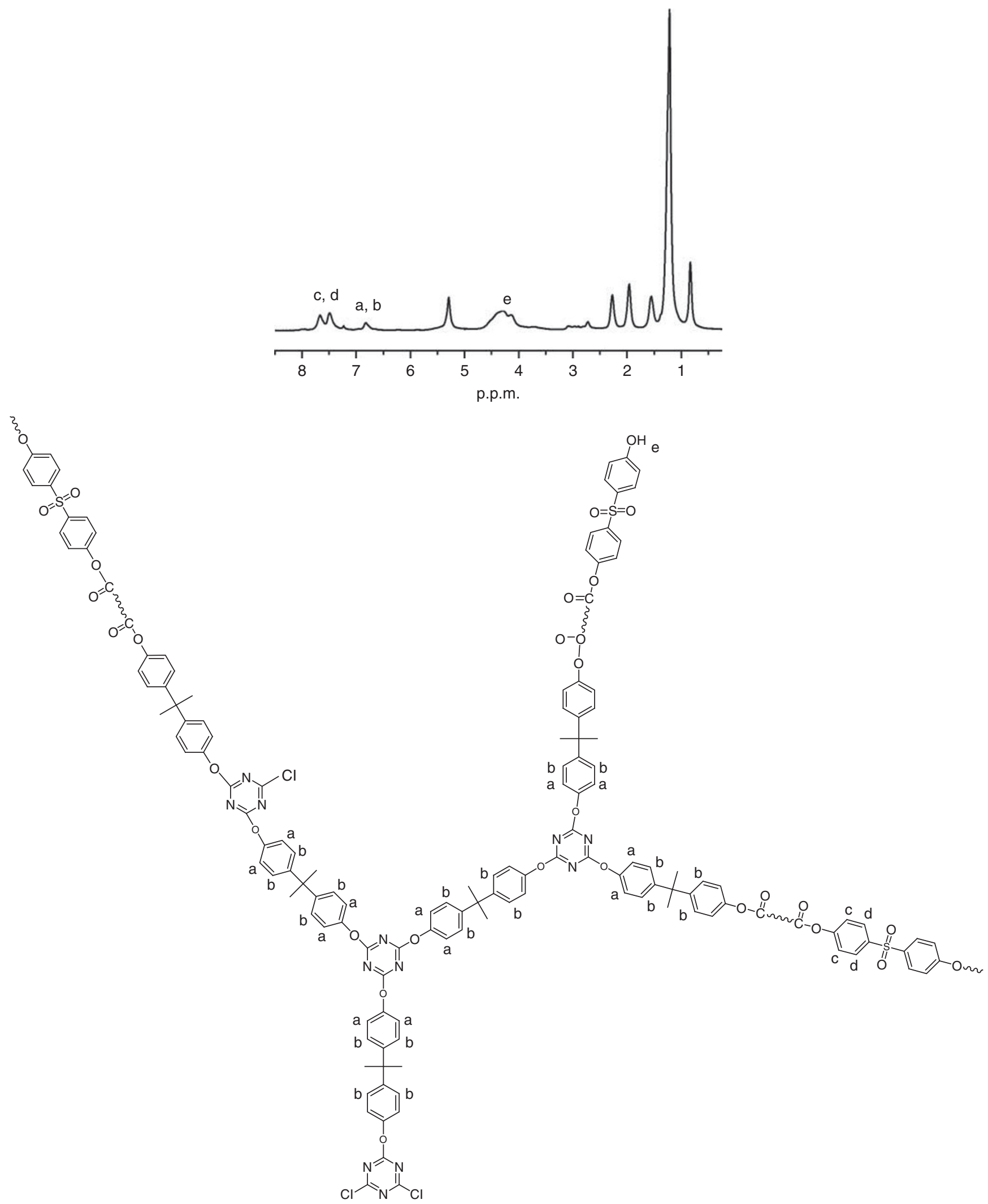

Figure $4{ }^{1} \mathrm{H}$ nuclear magnetic resonance spectrum of the vegetable oil-modified polyester with hyperbranched polyether core.

the polyester nanocomposites was significantly improved with the addition of OMMT (Table 2). The nanosize OMMT platelets had a vital role in this improvement. The nanofiller functioned as a crack stopper and forced the cracks to follow a tortuous pathway, which in turn resulted in high impact energy for the nanocomposite. The OMMT layers also interacted with the polyester as well as with the 


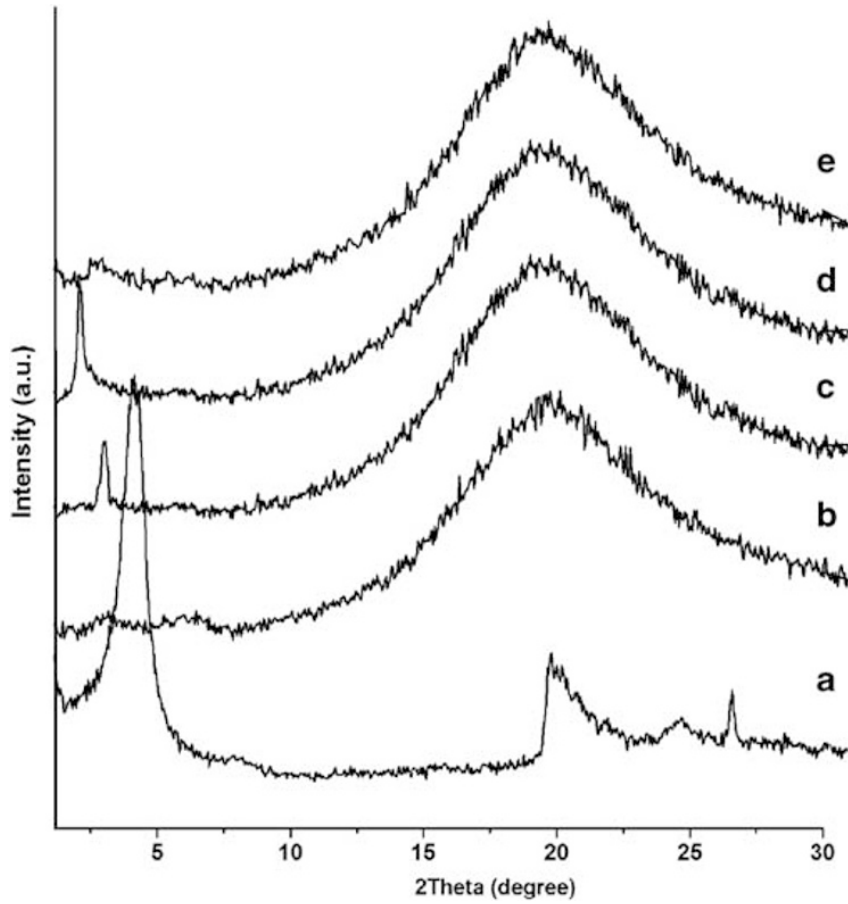

Figure 5 X-ray diffraction traces for (a) PE, (b) PNC1, (c) PNC2.5, (d) PNC5 and (e) OMMT.

Table 1 Interlayer spacing ( $d$-spacing) for the nanocomposites

\begin{tabular}{lccc}
\hline Nanocomposite code & $2 \theta\left(^{\circ}\right)$ & $\sin \theta$ & Interlayer spacing $(\mathrm{d}=\mathrm{n} \lambda / 2 \operatorname{Sin} \theta, \mathrm{nm})$ \\
\hline OMMT & 4.15 & 0.03621 & 2.12 \\
PNC0 & 19 & 0.16505 & - \\
PNC1 & 3 & 0.02617 & 2.94 \\
PNC2.5 & 2.12 & 0.01876 & 4.16 \\
PNC5 & 19 & 0.16505 & - \\
\hline
\end{tabular}

epoxy chains to restrict the chain mobility and increase the strength of the nanocomposite films.

From the curing-time measurements (Table 2), it was observed that curing time of the nanocomposites decreased with increasing OMMT loading from $0-5 \mathrm{wt} \%$. This decrease was due to the interaction of OMMT layers with the polyester chains, which restricted the mobility of the polymer segments. The high surface area of the OMMT assisted the crosslinking reaction through $\mathrm{H}$-bonding formed between hydroxyl groups of OMMT and hydroxyl/carboxyl groups of the polyester, along with the epoxy group of the epoxy resin (curing agent) and the amine groups of the poly(amido amine) hardener.

The chemical resistance of the cured vegetable oil-modified polyester with HBPE core nanocomposites was tested in various chemical environments (Table 3) for 15 days at room temperature. The alkali resistance of the prepared polyester resin was poor because of the presence of the hydrolyzable ester group. This poor alkali resistance was improved after the resin was formed as nanocomposites with $0-5 \mathrm{wt} \%$ OMMT. The fully dispersed OMMT layers in the nanocomposites functioned as a barrier by increasing the mean effective path of the alkali medium to enter the polyester matrix, which evaded the alkali absorption of the polymer system. The improvement of chemical resistance toward all other media was also found in the nano- composites with $0-5 \mathrm{wt} \%$ OMMT loadings. This improvement was due to the interaction of OMMT layers with the polyester chains that resulted in more compact and crosslinked structures in the nanocomposites. The permeability of the nanocomposites was also reduced by delaminated OMMT layers, which produced a tortuous pathway for diffusion. Therefore, the different ions or species present in different media could not penetrate the surface easily, which caused the chemical resistance to increase after the nanocomposite was formed.

\section{Rheological studies}

The rheological studies of the vegetable oil-modified polyester resin with HBPE core and its nanocomposites were performed on a controlled strain rheometer equipped with parallel plate geometry. Figure $8 \mathrm{a}$ showed the effect of time on shear viscosity of all nanocomposites. The shear viscosity remained nearly constant with time. The overall viscosities of all nanocomposites were higher than that of the pristine polyester. With the increase in OMMT loading (from 0 to $5 \mathrm{wt} \%$ ), the shear viscosity increases. This increase in shear viscosity was due to the intercalation/partial exfoliation of polyester chain molecules into the OMMT interlayer. The high level of interaction between the -OH groups of the OMMT with the polymer chains was also a contributing factor to this increase. Because of partial exfoliation, a more highly exposed OMMT surface was produced and this resulted in more interaction between the polymer chains and OMMT. Consequently, the interaction partially arrested the segmental movements of the pristine polymer. As the OMMT loadings increased, more and more polymer chains diffused into the relatively empty gallery of the silicate. As a result, the viscosity of the nanocomposite system gradually increased.

The behavior of shear viscosity with respect to temperature of the pristine polyester and its nanocomposites is shown in Figure 8b. The shear viscosity decreased with the increase in temperature. This was due to the low intermolecular attraction among OMMT and polyester chains and increased kinetic energy of the molecules present in the nanocomposites. The dependence of steady shear viscosity on shear rate of the polyester nanocomposites is shown in Figure 8c. The shear viscosity of the nanocomposites with $0-5 \mathrm{wt} \%$ loadings showed significantly higher shear viscosities than the pure polyester system. The polymers with high molecular weights have a tendency to entangle their neighboring macromolecules into their three-dimensional network at low shear rates. However, the molecules are usually oriented in the shear direction during the shear process by entangling to an extent that lowers their flow resistance. This behavior is attributed to the physical jamming or percolation of randomly distributed silicate layers. ${ }^{16}$ The OMMT layers, especially the intercalated or exfoliated layers, could support this orientation by aligning their rigid crystalline lamellar structures toward the direction of flow at higher shear rates, as similarly reported by other researchers. ${ }^{26,27}$ The high shear viscosity was attributed to the strong interaction between dispersed OMMT layers and polymer chains at low shear rates. Furthermore, the interaction between OMMT layers became stronger at higher OMMT contents as the distance between layers became smaller. Consequently, the restricted local environment of the OMMT layers limited the ability of the polymer chains to relax. The high shear rates caused a breakdown in the network structure and more platelets indeed aligned in parallel. The OMMT silicate layers then oriented in the flow direction and contributed to the shearthinning behavior in the nanocomposites. Because of this shearthinning behavior, the nanocomposites could be processed in the melt state using conventional equipment available in a manufacturing 

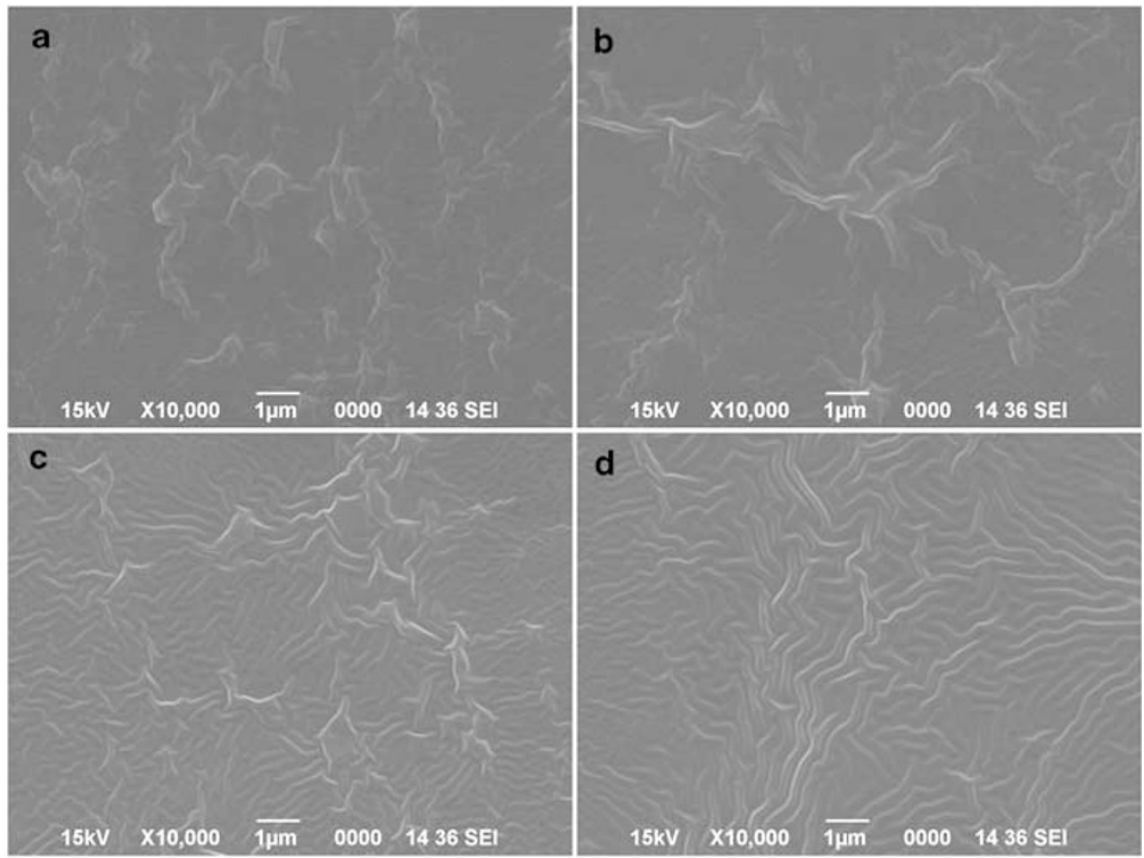

Figure 6 Scanning electron microscopy micrographs for (a) PE, (b) PNC1, (c) PNC2.5 and (d) PNC5.

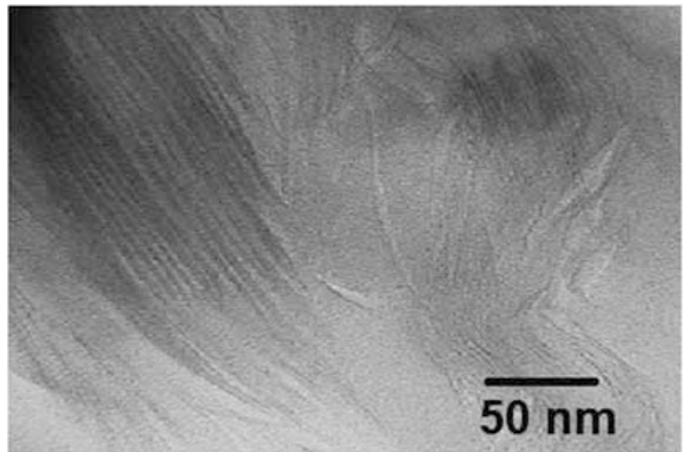

Figure 7 A representative transmission electron microscopy micrograph for PNC5.

line. Pronounced shear thinning has also been found to be a characteristic feature of truly nanodispersed composites.

\section{Thermal properties}

The thermal degradation behaviors of the vegetable oil-modified polyester with HBPE core and its nanocomposites are depicted in Figure 9. The degradation temperature of the virgin matrix increased with the incorporation of nanoclays. The improvement in the degradation temperature was due to the homogenous dispersion of the silicate OMMT nanoplatelets in the polyester matrix. The thermal decomposition of virgin polyester started at a temperature of $300^{\circ} \mathrm{C}$ and nearly completed at $650{ }^{\circ} \mathrm{C}$. Conversely, in the case of nanocomposites PNC1, PNC2.5 and PNC5, the onset of thermal decomposition was observed at 319,329 and $337^{\circ} \mathrm{C}$, respectively. Thus, the degradation of the polyester took place at a higher temperature in the presence of OMMT. An increase of $37^{\circ} \mathrm{C}$ in thermal degradation temperature was observed for the PNC5 sample compared with the pristine polyester. This increase in the thermal stability could be explained by the presence of a diffusion effect, which limited the emission of
Table 2 Performance characteristics of pristine polyester and its nanocomposites

\begin{tabular}{lcccc}
\hline Property & $P E$ & PNC1 & PNC2.5 & PNC5 \\
\hline Gloss at 60 & 83 & 93 & 96 & 98 \\
Scratch hardness (kg) & 8 & 8.5 & 10 & 10 \\
Impact resistance (cm) & 100 & $>100$ & $>100$ & $>100$ \\
Tensile strength ( $\mathrm{mm}^{-2}$ ) & 5.46 & 7.87 & 8.99 & 11.18 \\
Elongation at break (\%) & 102.21 & 89.4 & 57.05 & 47.75 \\
Curing time (min at $\left.120^{\circ} \mathrm{C}\right)$ & 80 & 60 & 50 & 45 \\
Swelling (\%) in xylene & 35.26 & 33.16 & 32 & 30.5 \\
\hline
\end{tabular}

Table 3 Chemical resistances of the pristine polyester and its nanocomposites in different chemical media

\begin{tabular}{lllll}
\hline & \multicolumn{4}{c}{ Weight loss (\%) } \\
\cline { 2 - 5 } Medium & $P E$ & PNC1 & PNC2.5 & PNC5 \\
\hline 5 wt\% Aqueous NaOH & 5.12 & 1.42 & 0.80 & 0.30 \\
25 wt\% Aqueous HCl & 0.50 & 0.26 & 0.12 & 0.09 \\
25 wt\% Aqueous $\mathrm{NaCl}$ & 0.40 & 0.18 & 0.13 & 0.08 \\
Distilled water & 0.08 & 0.06 & 0.05 & 0.01 \\
\hline
\end{tabular}

gaseous degradation products and resulted in increased thermal stability. ${ }^{28}$ Therefore, the OMMT enhanced thermal stability by functioning as a mass-transport barrier to the volatile product generated during decomposition as a result of decreasing permeability. ${ }^{29}$ Again, the amount of char residue formed in the nanocomposites increased with the incorporation of OMMT in the polyester matrix. 

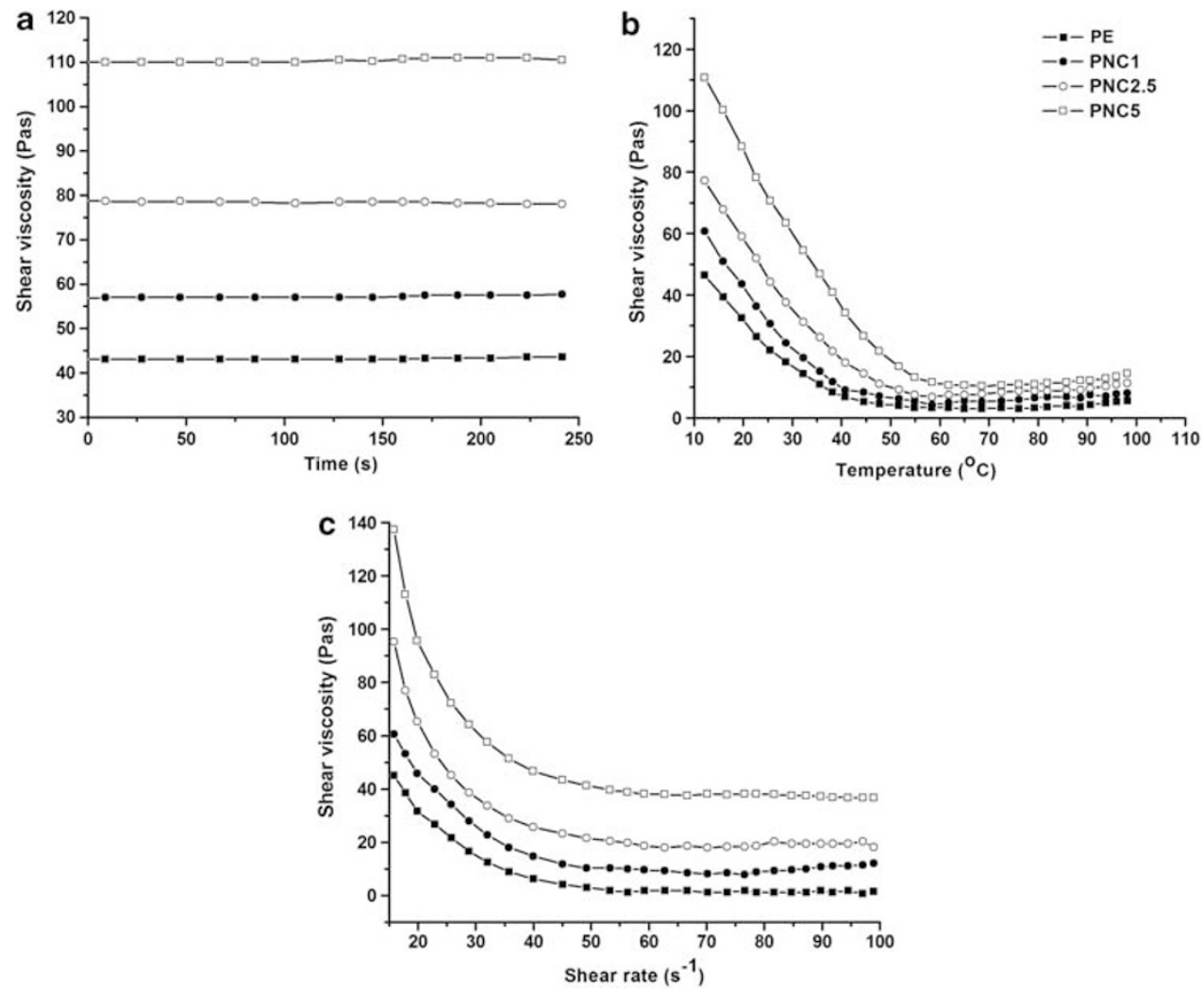

Figure 8 Variation of shear viscosity of polyester resin/clay nanocomposites versus (a) time at constant stress and temperature, (b) temperature under constant stress and (c) shear rate under constant temperature.

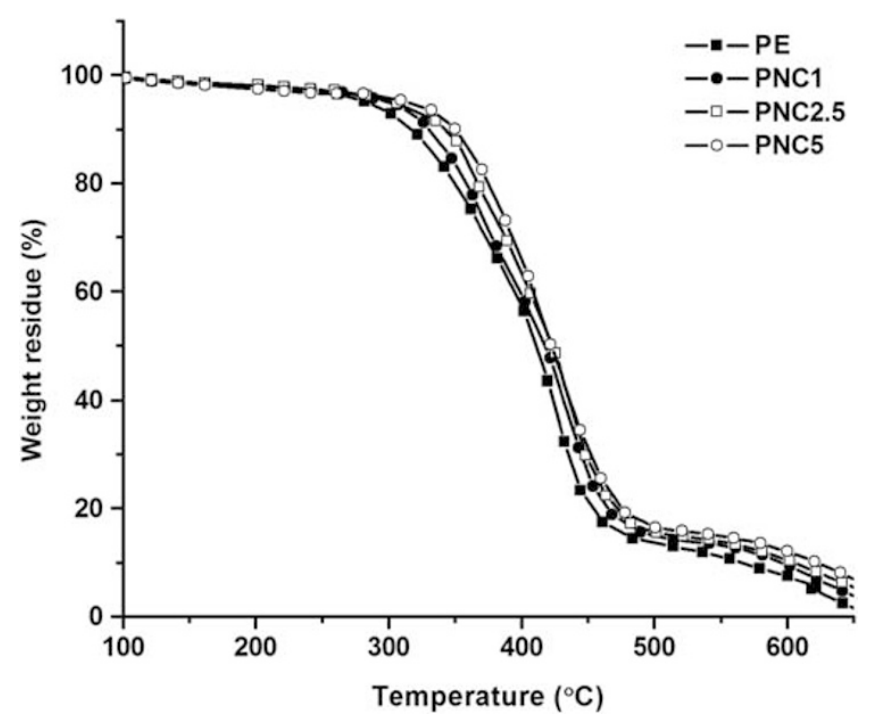

Figure 9 Thermogravimetric analysis thermographs for the pristine polyester and its nanocomposites.

\section{CONCLUSIONS}

Vegetable oil-modified polyester resin with HBPE core was synthesized and characterized in detail. The same polymer-based nanocomposites with OMMT as a nanofiller at $0-5 \mathrm{wt} \%$ loadings were prepared ex situ using a solution technique involving mechanical shearing force and ultrasonication. XRD, SEM and TEM studies revealed the formation of partially exfoliated nanocomposites. Properties such as tensile strength, impact resistance, hardness and thermostability improved when the pristine polyester system was formed into a nanocomposite. Thus, the study revealed the potentiality of the prepared vegetable oilmodified polyester with HBPE core nanocomposites as an advanced surface-coating material.

\section{ACKNOWLEDGEMENTS}

We are grateful for the assistance on the research project provided by DRDO, India, through grant ERIP/ER/0403490/M/01/962, dated 14 May 2007. We also express our gratitude to the Department of Physics, Tezpur University and North-Eastern Hills University, Shillong, for their help with XRD and SEM analyses and TEM analyses, respectively.

1 Haq, M., Burgueño, R., Mohanty, A. K. \& Misra, M. Hybrid bio-based composites from blends of unsaturated polyester and soybean oil reinforced with nanoclay and natural fibers. Compos. Sci. Technol. 68, 3344-3351 (2008).

2 Raquez, J. M., Deléglise, M., Lacrampe, M. F. \& Krawczak, P. Thermosetting biomaterials derived from renewable resources: a critical review. Prog. Polym. Sci. 35, 487-509 (2010).

3 Uyama, H., Kuwabara, M., Tsujimoto, T., Nakano, M., Usuki, A. \& Kobayashi, S. Green nanocomposite from renewable resources: plant oil-clay hybrid materials. Chem. Mater. $15,2492-2494$ (2003).

4 Tsujimoto, T., Uyama, H. \& Kobayashi, S. Synthesis of high-performance green nanocomposites from renewable natural oils. Polym. Degrad. Stab. 95, 1399-1405 (2010).

5 Samman, M. A., Radke, W., Khalyavina, A. \& Lederer, A. Retention behavior of linear, branched, and hyperbranched polyesters in interaction liquid chromatography. Macromolecules 43, 3215-3220 (2010).

6 Bao, C. L., Wang, L. S. \& Zhang, A. Q. Synthesis and properties of waterborne hyperbranched aliphatic polyester clear coats. J. Taiwan. Inst. Chem. Eng. 40, 174-179 (2009). 
7 van Benthem, R.A.T.M. Novel hyperbranched resins for coating applications. Prog. Org. Coat. 40, 203-214 (2000).

8 Ikhuoria, E. U., Aigbodion, A. I. \& Okieimen, F. E. Preparation and characterisation of water-reducible alkyds with fumarized rubber seed oil. Prog. Org. Coat. 52, 238-240 (2005).

9 Uyama, H., Kuwabara, M., Tsujimoto, T. \& Kobayashi, S. Enzymatic synthesis and curing of biodegradable epoxide-containing polyesters from renewable resources. Biomacromolecules 4, 211-215 (2003).

10 Ahmad, S., Ashraf, S. M. \& Zafar, F. Development of linseed oil based polyesteramide without organic solvent at lower temperature. J. Appl. Polym. Sci. 104, 1143-1148 (2007).

11 Konwar, U., Karak, N. \& Mandal, M. Mesua ferrea L. seed oil based highly thermostable and biodegradable polyester/clay nanocomposites. Polym. Degrad. Stab. 94, 22212230 (2009).

12 Mahapatra, S. S. \& Karak, N. Fluorescent hyperbranched polyamine with s-triazine: synthesis, characterization and properties evaluation. Polym. J. 41, 20-25 (2009).

13 Konwar, U., Karak, N. \& Mandal, M. Vegetable oil based highly branched polyester/clay silver nanocomposites as antimicrobial surface coating materials. Prog. Org. Coat. 68, 265-273 (2010).

14 Dutta, S., Karak, N., Saikia, J. P. \& Konwar, B. K. Biocompatible epoxy modified bio-based polyurethane nanocomposites: mechanical property, cytotoxicity and biodegradation. Bioresour. Technol. 100, 6391-6397 (2009).

15 Konwar, U. \& Karak, N. Mesua ferrea I. Seed oil-based highly branched polyester resins. Polym. Plast. Technol. Eng. 48, 970-975 (2009).

16 Pavlidou, S. \& Papaspyrides, C. D. A review on polymer-layered silicate nanocomposites. Prog. Polym. Sci. 33, 1119-1198 (2008).

17 Wang, X., Gao, Y., Mao, K., Xue, G., Chen, T., Zhu, J., Li, B., Sun, P., Jin, Q., Ding, D. \& Shi, A. C. Unusual rheological behavior of liquid polybutadiene rubber/clay nanocomposite gels: the role of polymer-clay interaction, clay exfoliation, and clay orientation and disorientation. Macromolecules 39, 6653-6660 (2006).

18 Haq, M., Burgueño, R., Mohanty, A. K. \& Misra, M. Bio-based polymer nanocomposites from UPE/EML blends and nanoclay: Development, experimental characterization and limits to synergistic performance. Composites: Part A Appl. Sci. Manufacture 42, 41-49 (2011).

19 Bal, A., Guclu, G., Acar, I. \& Iyim, T. B. Effects of urea formaldehyde resin to film properties of alkyd-melamine formaldehyde resins containing organo clay. Prog. Org. Coat. 68, 363-365 (2010).

20 Lu, J., Hong, C. K. \& Wool, R. P. Bio-based nanocomposites from functionalized plant oils and layered silicate. J. Polym. Sci. Part B: Polym. Phys. 42, 1441-1450 (2004).

21 Borah, J. \& Karak, N. Synthesis and characterization of a novel hyperbranched polyether. Polym. Int. 53, 2026-2030 (2004).

22 Karak, N., Roy, B. \& Voit, B. s-Triazine-based hyperbranched polyethers: synthesis, characterization, and properties. J. Polym. Sci. Part A: Polym. Chem. 48, 3994-4004 (2010).

23 Yu, L., Petinakis, S., Dean, K., Bilyk, A. \& Wu, D. Green polymeric blends and composites from renewable resources. Macromol. Symp. 249-250, 535-539 (2007).

24 Ahn, Y. H. \& Chang, J. H. Thermotropic liquid crystalline polyester nanocomposites via in situ intercalation polycondensation. Polym. Adv. Technol. 19, 1479-1485 (2008).

25 Maji, P. K., Guchhait, P. K. \& Bhowmick, A. K. Effect of the microstructure of a hyperbranched polymer and nanoclay loading on the morphology and properties of novel polyurethane nanocomposites. ACS Appl. Mater. Interfaces 1, 289-300 (2009).

26 Kim, T. H., Jang, L. W., Lee, D. C., Choi, H. J. \& Jhon, M. S. Synthesis and rheology of intercalated polystyrene/na ${ }^{+}$-montmorillonite nanocomposites. Macromol. Rapid. Commun. 23, 191-195 (2002).

27 Yỳlmaz, O., Cheaburu, C. N., Gülümser, G. \& Vasile, C. Rheological behaviour of acrylate/montmorillonite nanocomposite latexes and their application in leather finishing as binders. Prog. Org. Coat. 70, 52-58 (2011).

28 Duquesne, S., Jama, C., Bras, M. L., Delobel, R., Recourt, P. \& Gloaguen, J. M. Elaboration of EVA-nanoclay systems-characterization, thermal behaviour and fire performance. Compos. Sci. Technol. 63, 1141-1148 (2003).

29 Alexandre, M. \& Dubois, P. Polymer-layered silicate nanocomposites: preparation, properties and uses of a new class of materials. Mater. Sci. Eng. 28, 1-63 (2000). 